\title{
Desenvolvimento de um Instrumento para o Registro da Experiência Docente no Uso de Laboratórios Virtuais
}

\author{
Victor Vieira ${ }^{1,2}$, Bruno Gadelha1 \\ ${ }^{1}$ Instituto de Computação - Universidade Federal do Amazonas (UFAM) \\ 69.077-000 - Manaus - AM - Brasil
}

${ }^{2}$ Grupo de Informática para Pesquisa em Computação - Instituto Federal do Acre (IFAC) 69.903-068 - Acre - AC - Brasil

victor.vieira@ifac.edu.br, bruno@icomp.ufam.edu.br

\begin{abstract}
Virtual laboratories are educational software that allows the simulation of activities of real laboratories, motivating students and reducing costs and dangers. Many usage information is lost after an activity with a virtual lab. This paper presents an instrument for the registration of teachers experience in the use of these laboratories. As a result, in addition to the instrument, there is a web tool that reflects the experiences of use, composing an infrastructure capable of making the registered information, generally discarded, useful to assist in the selection of virtual laboratories.
\end{abstract}

Resumo. Laboratórios virtuais são softwares educativos que permitem a simulação de atividades de laboratórios reais, motivando os alunos e reduzindo custos e perigos. Muitas informações sobre o uso são perdidas após uma atividade com um laboratório virtual. Este trabalho apresenta um instrumento para o registro da experiência docente no uso desses laboratórios. Como resultado, além do instrumento, tem-se a ferramenta VLEx, que reflete as experiências de uso, compondo uma infraestrutura capaz de tornar as informações registradas, geralmente descartadas, úteis para auxiliar na seleção de laboratórios virtuais.

\section{Introdução}

A evolução tecnológica das últimas décadas gerou diversas possibilidades de tornar o aprendizado mais interativo. Isso ocorreu motivado pelo perfil atual dos alunos, que cada vez mais cedo se tornam usuários das tecnologias digitais. Nesse cenário, os professores possuem como recurso ferramentas como os Softwares Educativos (SEs), com uma abordagem de ensino diferente da tradicional sala de aula [Godoi e Padovani, 2009].

Uma categoria de Software Educativo (SE) que motiva os estudantes nas atividades realizadas e apresenta bons resultados com relação à aprendizagem é a dos Laboratórios Virtuais (LVs) [Koh, 2017]. São programas que possibilitam aos alunos, através de um dispositivo tecnológico, realizarem experimentos ou simularem atividades que ocorrem em ambientes físicos sem a necessidade de estarem em um laboratório real. O uso dos LVs também traz benefícios por possibilitar a realização de atividades geralmente caras ou perigosas com um baixo custo e em um ambiente seguro, e é classificado como um fator motivador em comparação com a instrução em sala de aula e utilização de laboratórios remotos [Diwakar et al., 2016; Koh, 2017]. 
VII Congresso Brasileiro de Informática na Educação (CBIE 2018)

Anais do XXIX Simpósio Brasileiro de Informática na Educação (SBIE 2018)

Apesar dos LVs serem uma alternativa, os professores se deparam com um problema quando resolvem utilizá-los: a escolha do software [Godoi e Padovani, 2009; Frerich et al., 2014]. Um dos critérios que podem ser utilizados para essa escolha é a experiência de outros professores no uso. Porém, na maioria das vezes, nenhuma informação fica registrada sobre como o uso ocorreu. Os registros de experiências de uso são importantes para auxiliar professores no processo de seleção de LVs por fornecerem informações sobre a utilização deles em diferentes contextos, indicando metodologias e características mais adequadas.

Os trabalhos de Diwakar et al., [2016], Lucena et al. [2013], Yalcinap e Emiroglu [2012], Brito e Cunha [2012] e Seniow et al. [2010] dão suporte ao registro de experiências com LVs, mas partem dos pontos de vista dos alunos, dos desenvolvedores, ou, até mesmo, de informações do próprio sistema. Além disso, as metodologias utilizadas para os registros nesses trabalhos não são bem descritas e as tecnologias para tal não estão disponíveis. Dessa forma, nenhum dos trabalhos relacionados a este artigo apresenta uma tecnologia capaz de registrar, na perspectiva do docente: a) o que, de fato, aconteceu com relação ao uso do laboratório virtual; b) que metodologia foi utilizada; c) como os alunos acessaram; d) que tipo de suporte foi fornecido; e e) observações adicionais relevantes. Considera-se que esses fatores são mais importantes na hora de decidir qual LV utilizar.

Este trabalho trata de um instrumento para registro da experiência docente no uso de laboratórios virtuais. $\mathrm{O}$ instrumento passou por duas versões avaliadas/validadas até chegar à terceira e última, que gerou uma ferramenta que reflete, do ponto de vista do professor, cada experiência usando um laboratório virtual (LV).

O restante do texto está organizado da seguinte forma: a Seção 2 apresenta a metodologia da pesquisa, descrevendo cada etapa; a Seção 3 traz os resultados e discussões, classificados de acordo com a evolução do instrumento; na Seção 4, são apresentadas as considerações finais, com objetivos alcançados, contribuição da pesquisa e expectativas para trabalhos futuros.

\section{Metodologia}

Esta pesquisa aconteceu de acordo com o protocolo ilustrado na Figura 1, que define as seguintes etapas: $1^{\text {a }}$ ) desenvolvimento da primeira versão do instrumento a partir de buscas iniciais; $2^{a}$ ) estudo piloto com a primeira versão; $3^{a}$ ) Revisão Sistemática da Literatura (RSL); $4^{\text {a }}$ ) desenvolvimento da segunda versão do instrumento; $5^{\mathrm{a}}$ ) avaliação de especialistas; e $6^{\text {a }}$ ) desenvolvimento da terceira versão do instrumento e desenvolvimento da ferramenta para a exibição dos registros realizados. Essas etapas são descritas a seguir.

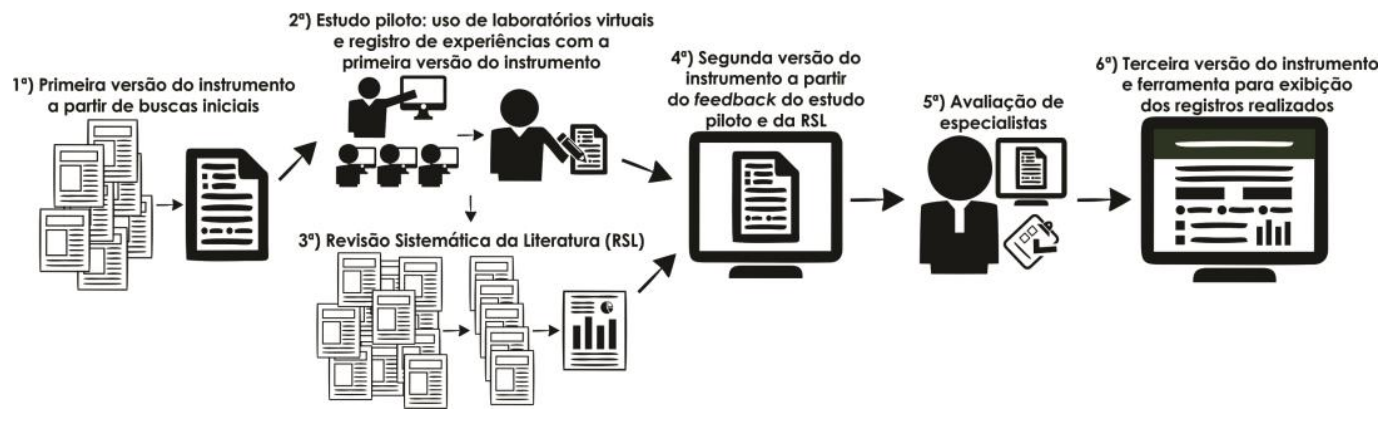

Figura 1. Etapas da pesquisa 
VII Congresso Brasileiro de Informática na Educação (CBIE 2018)

Anais do XXIX Simpósio Brasileiro de Informática na Educação (SBIE 2018)

Inicialmente, foram realizadas buscas iniciais abordando o problema da escolha de LVs. Nessas buscas, foram utilizados os termos "seleção", "utilização" e "laboratórios virtuais" para encontrar trabalhos mais recentes no Google Acadêmico e Anais da Revista Brasileira de Informática na Educação (RBIE) e do Simpósio Brasileiro de Informática na Educação (SBIE), selecionados a partir da leitura dos resumos em um único filtro, e analisados completamente, em seguida. Verificou-se que a utilização de informações de uma experiência de uso para auxiliar na escolha de um software era incomum, bem como não foi encontrada uma tecnologia ou procedimento bem estruturado para registrar tais experiências.

A partir desse problema e dos resultados encontrados, foi desenvolvida a primeira versão do instrumento que realiza o registro da experiência docente no uso de um LV. Trata-se de um questionário, escolhido por ser uma tecnologia fácil de ser ministrada e que facilita a análise dos dados [Lakatos e Marconi, 2017]. Essa primeira versão foi utilizada em dois casos de uso de LVs em disciplinas de Manutenção de Computadores no Instituto Federal do Acre (IFAC), como um estudo piloto. Ao final do estudo piloto, verificou-se o potencial do instrumento de registrar, de fato, como um LV foi utilizado em uma turma e o potencial dos registros para dar suporte à escolha de laboratórios por outros professores.

Em seguida, foi realizada uma RSL onde se obteve uma visão geral das tecnologias existentes para o registro da experiência de uso de LV publicadas a partir de 2010, com objetivo de melhorar a primeira versão. A data foi definida após as buscas iniciais resultarem em trabalhos a partir dela, indicando que o tema é recente, não havendo sido encontrada nenhuma revisão ou mapeamento da literatura sobre o assunto que pudesse ser utilizada como ponto de partida. A RSL foi realizada de acordo com as diretrizes fornecidas por Kitchenham e Chartes [2007], com as buscas ocorrendo nas bibliotecas digitais da Scopus, Engineering Village, RBIE, SBIE e no Google Acadêmico, e pode ser acessada no Relatório Técnico RT-GSI-2018-002 [Vieira e Gadelha, 2018b]. A partir da revisão, o instrumento foi melhorado para a segunda versão.

A segunda versão do instrumento foi submetida à avaliação de especialistas, professores com experiência em LVs, através de um questionário. As respostas dessa avaliação foram analisadas quantitativamente e qualitativamente. $\mathrm{O}$ instrumento foi remodelado a partir das opiniões desses especialistas para a terceira versão. Uma ferramenta denominada VLEx foi desenvolvida para exibir as informações dos registros realizados na terceira versão do instrumento. Consiste em uma página onde os resultados são agrupados por LV e cada experiência ainda pode ser visualizada individualmente.

\section{Instrumento para Registro de Experiência Docente no Uso de LV}

A seguir, é descrito como o instrumento para registrar experiências de professores com LVs evoluiu, desde sua primeira versão, passando pelo estudo piloto, RSL, segunda versão, avaliação dos especialistas, terceira versão e ferramenta para a exibição dos registros. 
VII Congresso Brasileiro de Informática na Educação (CBIE 2018)

Anais do XXIX Simpósio Brasileiro de Informática na Educação (SBIE 2018)

\subsection{Primeira Versão}

A primeira versão do instrumento consiste em um questionário com 18 questões, doze fechadas e seis abertas, e pode ser visualizada no Relatório Técnico RT-GSI-2018-001 [Vieira e Gadelha, 2018a]. Para sua construção, levou-se em consideração trabalhos das buscas iniciais, como os de Lucena et al. [2013,] Diwakar et al. [2016] e Nunes et al. [2017], com pontos considerados necessários para se obter uma visão ampla da adoção como: contexto, como foi utilizado, suporte fornecido, metodologia proposta para a atividade e observações adicionais. As questões foram classificadas em relacionadas ao uso, ao LV, aos alunos e às observações realizadas pelo professor.

\subsubsection{Estudo Piloto}

No estudo piloto, dois professores de disciplinas de Manutenção de Computadores utilizaram LVs com suas turmas no IFAC e, em seguida, registraram as experiências com a primeira versão do instrumento. Com relação à atividade de utilização dos LVs, os professores perceberam uma melhoria na motivação dos alunos e classificaram os laboratórios como uma estratégia importante para situações envolvendo custo ou risco como, por exemplo, o de danificar componentes de hardware por mau uso.

As opiniões dos professores sobre a primeira versão do instrumento no estudo piloto mostraram que eles o consideraram fácil de ser preenchido, principalmente por contar com a maioria das questões fechadas, e como uma estratégia válida para aproveitar as informações sobre como ocorreu o uso. Ainda sugeriram a adição de textos de ajuda para cada questão, visto que tiveram dificuldades em entender o que estava sendo perguntado em algumas. Também relataram que o preenchimento seria mais fácil se fosse realizado em um questionário eletrônico, já que receberam as questões em um documento de texto, para serem respondidas à mão ou no editor de textos. No geral, o feedback foi classificado como positivo e todas as sugestões foram consideradas para a segunda versão do instrumento.

\subsection{Segunda Versão}

O feedback dos professores no estudo piloto apontou para a necessidade de melhorias no instrumento de registro de experiências com os LVs. Decidiu-se, então, realizar uma RSL com o objetivo de verificar quais tecnologias existem para registrar experiências com uso de LVs e como funcionam. Como resultado, a segunda versão do instrumento foi desenvolvida, com características das tecnologias existentes e descobertas realizadas.

As questões foram organizadas de modo a esclarecer sobre oito tópicos que foram considerados importantes: cenário, suporte, acesso, usabilidade, motivação, simulação, metodologia e geral. Essa divisão foi pensada para agrupar questões que abordam pontos comuns e criar um roteiro lógico para o preenchimento, assim como aconteceu na primeira versão, onde a classificação seguiu outro padrão já citado. A seguir, são discutidos cada um dos tópicos.

As questões relacionadas ao cenário consistem em itens de identificação, em sua maioria, encontrados em todos os trabalhos que descrevem o uso de SEs, como o nome do software, quantidade de alunos e disciplina em que foi utilizado, entre outros. As questões sobre suporte dizem respeito ao suporte fornecido aos alunos antes e durante a 
VII Congresso Brasileiro de Informática na Educação (CBIE 2018)

Anais do XXIX Simpósio Brasileiro de Informática na Educação (SBIE 2018)

utilização do LV e foram baseadas nos trabalhos de Lucena et al. [2013] e Ramírez et al. [2017].

As questões sobre acesso dizem respeito aos dispositivos utilizados, à facilidade de instalação nesses dispositivos e à forma de uso do LV, que poderia ser individual ou em grupo. A escolha da forma de uso, segundo Diwakar et al. [2016], depende da estrutura existente e da metodologia utilizada pelo professor. Trabalhos como os de Yalcinap e Emiroglu [2012] e Brito e Cunha [2012] apontam que a facilidade de acesso é determinante para a escolha de um LV.

No tópico usabilidade, uma questão diz respeito às dificuldades percebidas pelo professor no uso do LV pelos alunos. No estudo realizado por Kalyvioti e Mikropoulos [2013], os autores relacionaram a facilidade de uso de um LV, apontada pelos estudantes, com melhores resultados na aprendizagem e motivação. A indicação de que houve ou não dificuldades no uso por parte dos alunos é considerada relevante para a seleção de um software e, até mesmo, para avaliá-lo.

No tópico motivação, uma questão aborda se, na percepção do professor, os alunos estiveram motivados a desenvolver a atividade proposta. Tal tópico é relevante dado que atividades com alunos motivados tendem a melhores resultados, mesmo com aplicações bastante simples, como reportado por Martins et al. [2012]. Outros trabalhos ainda destacam o sucesso dos LVs com relação à motivação, como os de Brito e Cunha [2012], Diwakar et al. [2016] e Koh [2017].

As questões sobre simulação são as que mais estão relacionadas aos LVs, por abordarem o nível de fidelidade da atividade, em comparação com como ocorre em um laboratório real, e a preparação fornecida. Nesse sentido, os trabalhos de Diwakar et al. [2016], Koh [2017] e Ramírez et al. [2017] destacam resultados positivos na prática em ambiente real associados ao uso de LVs anteriormente. Dessa forma, professores tendem a selecionar os que simulam melhor suas atividades.

No tópico metodologia, uma questão aborda como o LV foi utilizado, ou seja, a metodologia definida pelo professor. Para Seniow et al. [2010], a metodologia de uso dos LVs tem um grande potencial para melhorar o aprendizado dos alunos. Essa informação é útil, principalmente, para professores sem experiência no uso desses SEs, pois podem visualizar metodologias em experiências com resultados positivos, adaptálas e utilizá-las.

O último tópico agrupa pontos positivos, negativos e observações adicionais sobre a atividade, e é nomeado de geral. Os pontos positivos são indicativos de acerto na utilização de um LV. Os negativos indicam problemas, falhas ou dificuldades que podem ser observadas para que não ocorram em outras experiências. As observações adicionais ainda contemplam informações relevantes que não couberam como resposta a nenhuma outra questão e são tão importantes para auxiliar na seleção de um LV como as demais.

Essa versão do instrumento contém 31 questões, entre abertas, fechadas e em escala Likert de cinco pontos, foi disponibilizada como Formulário do Google e pode ser visualizada no Relatório Técnico RT-GSI-2018-003 [Vieira e Gadelha, 2018c]. Depois de concluída, a versão foi submetida à avaliação de especialistas, descrita a seguir. 
VII Congresso Brasileiro de Informática na Educação (CBIE 2018)

Anais do XXIX Simpósio Brasileiro de Informática na Educação (SBIE 2018)

\subsubsection{Avaliação de Especialistas}

Com intuito de avaliar o instrumento gerado, a segunda versão foi submetida à análise de sete especialistas. Eles foram escolhidos por trabalharem com essa categoria de SE e possuírem experiência mínima de cinco anos em docência. As opiniões foram emitidas através de respostas a um questionário, indicando: 1) se as informações providas através do instrumento ajudam na escolha de um LV; 2) se as perguntas eram suficientes para descrever todos os aspectos relevantes do uso do LV em uma atividade; 3 e 4) pontos positivos e negativos do instrumento; e 5) sugestões de melhorias. A Tabela 1 apresenta os resultados quantitativos das duas questões em escala Likert de cinto pontos (de discordo totalmente a concordo totalmente). As demais questões são analisadas em seguida.

Tabela 1. Média e desvio padrão das questões em escala Likert para a avaliação da segunda versão do instrumento por especialistas

\begin{tabular}{|c|c|c|}
\hline Questões & Média & Desvio Padrão \\
\hline $\begin{array}{l}\text { 1) As informações providas pelos professores através do instrumento } \\
\text { ajudam na escolha de um laboratório virtual. }\end{array}$ & 3,28 & 1,70 \\
\hline $\begin{array}{l}\text { 2) As questões do instrumento são suficientes para descrever todos os } \\
\text { aspectos relevantes do uso do laboratório virtual em uma atividade. }\end{array}$ & 4,00 & 1,15 \\
\hline
\end{tabular}

O desvio padrão de 1,70 na primeira questão foi considerado elevado para a escala de cinco pontos com sete respondentes, e indicou que os especialistas não opinaram em consenso. Dois discordaram totalmente da afirmação, sugerindo que as informações oriundas do instrumento não ajudam na escolha de um LV. Contrários a essa opinião, a maioria concordou com a afirmação. Um opinou com indiferença. A média de 3,28 na questão indica uma tendência mínima do grupo de especialistas a concordar que a escolha de um LV pode ser auxiliada pelas informações obtidas através do instrumento.

$\mathrm{Na}$ segunda questão, a média de 4,00 indica que as questões do instrumento descrevem suficientemente os aspectos relevantes do uso de um LV, e o desvio padrão de 1,15 mostra que as opiniões não foram tão divergentes. Apenas um discordou da afirmação. Outro se mostrou indiferente e cinco especialistas concordaram. A realização da RSL, agregando características de outras tecnologias, foi fundamental para esse resultado, tornando o instrumento mais completo.

Os especialistas ainda listaram os pontos que consideraram positivos e negativos, além de alterações sugeridas no instrumento. Essas informações foram analisadas qualitativamente. As opiniões foram classificadas em categorias, geradas a partir das respostas, desconsiderando a relação entre as respostas e o enunciado de cada uma. Essa metodologia para análise qualitativa foi baseada no Método de Explicitação do Discurso Subjacente (MEDS), de Nicolaci-Da-Costa [2007]. As categorias que emergiram foram as seguintes, com a quantidade de ocorrências entre parênteses: clareza das questões (10); estrutura do questionário (7); conteúdo do questionário (6); e preenchimento do questionário (5).

Os comentários estiveram mais relacionados a problemas e sugestões de melhorias. Com relação à clareza, nove dos dez comentários criticaram a falta de clareza em alguns textos do instrumento. Com relação à estrutura, o instrumento foi considerado desorganizado em algumas partes e extenso. O conteúdo foi elogiado em cinco dos seis comentários, destacando a identificação de informações relevantes sobre 
VII Congresso Brasileiro de Informática na Educação (CBIE 2018)

Anais do XXIX Simpósio Brasileiro de Informática na Educação (SBIE 2018)

o uso de LVs. Com relação ao preenchimento, cinco dos seis comentários destacaram a dificuldade no preenchimento de algumas partes. Comentários como "as questões deveriam capturar mais objetivamente a percepção do professor visando o reuso do recurso, por exemplo: 'alunos foram auxiliados...' poderia ser 'alunos necessitaram/solicitaram auxilio..." " e "as perguntas poderiam ser reorganizadas para exibir as em escala Likert em um só bloco" reforçaram a necessidade de reformulação da escrita dos textos e rearranjo das questões.

Com as considerações realizadas pelos especialistas, a segunda versão do instrumento foi reformulada para a terceira versão, descrita a seguir.

\subsection{Terceira Versão}

A partir das análises realizadas pelos especialistas, o instrumento foi reformulado para a terceira versão, apresentada na Tabela 2 e disponível no Relatório Técnico RT-GSI2018-004 [Vieira e Gadelha, 2018d].

\section{Tabela 2. Terceira versão do instrumento para registro de experiência docente} no uso de laboratórios virtuais

\begin{tabular}{|c|c|c|c|}
\hline № & TÓPICO & TIPO & QUESTÃO \\
\hline 1 & \multirow{2}{*}{ Contato } & Aberta & Nome \\
\hline 2 & & Aberta & E-mail \\
\hline 3 & \multirow{9}{*}{ Contexto } & Aberta & Laboratório virtual \\
\hline 4 & & Aberta & Disponível em \\
\hline 5 & & Fechada & Área (todas as áreas do cnpq) \\
\hline 6 & & Aberta & Curso \\
\hline 7 & & Aberta & Disciplina \\
\hline 8 & & Fechada & Nível (fundamental/médio/técnico/superior/pós-graduação/outro) \\
\hline 9 & & Aberta & Quantidade de alunos \\
\hline 10 & & Aberta & Carga-horária da disciplina \\
\hline 11 & & Aberta & Carga-horária da prática com o laboratório virtual \\
\hline 12 & Metodologia & Aberta & Como você utilizou o laboratório virtual em sua disciplina? \\
\hline 13 & \multirow{4}{*}{ Suporte } & Likert & Antes da atividade, os alunos foram instruídos sobre como utilizar o laboratório virtual. \\
\hline 14 & & Likert & Antes da atividade, os alunos tiveram aula sobre o conteúdo abordado no laboratório virtual. \\
\hline 15 & & Likert & Durante a atividade, os alunos solicitaram auxílio na utilização do laboratório virtual. \\
\hline 16 & & Likert & Durante a atividade, os alunos solicitaram auxílio no(s) conceito(s) que estava(m) sendo desenvolvido(s). \\
\hline 17 & Usabilidade & Likert & Não percebi nenhum problema ou dificuldade no uso do laboratório virtual por parte dos alunos. \\
\hline 18 & Motivação & Likert & Percebi os alunos motivados a realizar a atividade no laboratório virtual. \\
\hline 19 & \multirow[t]{2}{*}{ Simulação } & Likert & $\begin{array}{l}\text { O laboratório virtual imita o experimento simulado exatamente como acontece em um laboratório real, em } \\
\text { relação aos materiais, métodos e resultados. }\end{array}$ \\
\hline 20 & & Likert & A atividade preparou os alunos para a prática em ambiente real. \\
\hline 21 & \multirow{5}{*}{ Acesso } & Likert & O laboratório virtual foi instalado/acessado facilmente. \\
\hline 22 & & Fechada & O laboratório virtual foi utilizado em qual(is) dispositivo(s)? (computador/smartphone/tablet/outro) \\
\hline 23 & & Aberta & Qual a justificativa para ter usado esse(s) dispositivo(s)? \\
\hline 24 & & Fechada & Os alunos tiveram acesso individual ao laboratório virtual? (sim/não/parcialmente) \\
\hline 25 & & Aberta & Qual a justificativa para essa forma de acesso? \\
\hline 26 & Desempenho & Aberta & Como você considera o aproveitamento dos alunos com o laboratório virtual? \\
\hline 27 & \multirow{3}{*}{ Geral } & Aberta & Quais os pontos positivos percebidos durante a atividade? \\
\hline 28 & & Aberta & Quais os pontos negativos percebidos durante a atividade? \\
\hline 29 & & Aberta & Descreva as observações adicionais. \\
\hline
\end{tabular}

As alterações realizadas consistem na reorganização, exclusão, inserção e alteração de questões a partir de uma análise criteriosa das recomendações, bem como a adição de dois novos tópicos: contato, que anteriormente fazia parte do contexto, com informações para que outros professores interessados em um registro disponibilizado possam contatar quem o fez; e desempenho, criado a partir de recomendações dos especialistas. O número de questões diminuiu de 31 para 29, mantendo questões abertas, fechadas e em escala Likert de cinco pontos. O rearranjo realizado possibilitou agrupar os itens em escala Likert e criar um roteiro lógico para o preenchimento seguindo as recomendações dos especialistas. 
VII Congresso Brasileiro de Informática na Educação (CBIE 2018)

Anais do XXIX Simpósio Brasileiro de Informática na Educação (SBIE 2018)

A partir da terceira versão do instrumento, disponibilizada como Formulário do Google, uma ferramenta para a exibição das informações dos registros foi desenvolvida e é apresentada a seguir.

\subsubsection{Ferramenta para a Exibição dos Registros}

Com o instrumento para registro de experiência docente no uso de LV remodelado para a terceira versão, foi desenvolvida uma ferramenta web denominada VLEx, que possibilita a visualização das experiências registradas. A motivação para desenvolvê-la foi melhorar a interação com as informações dos registros para explorar o potencial dessas informações no auxílio a professores na escolha de LVs.

Pelo fato de o instrumento ter sido disponibilizado como Formulário do Google, não seria possível a visualização das informações de um registro de forma organizada usando apenas a tecnologia do Google. Os dados seriam exibidos em uma planilha sem formatação, o que prejudicaria a análise. A VLEx organiza os dados e o acesso ao instrumento através de uma página web, facilitando a visualização e registro da experiência de uso.

A ferramenta pode ser visualizada na Figura 2 e está disponível em goo.gl/wWYAFH, permitindo ao usuário acessar a terceira versão do instrumento para registrar uma experiência no uso de um LV, visualizar LVs que contém registros realizados, obter uma visão geral de um LV através dos registros realizados nele e visualizar todas as informações de um registro específico.

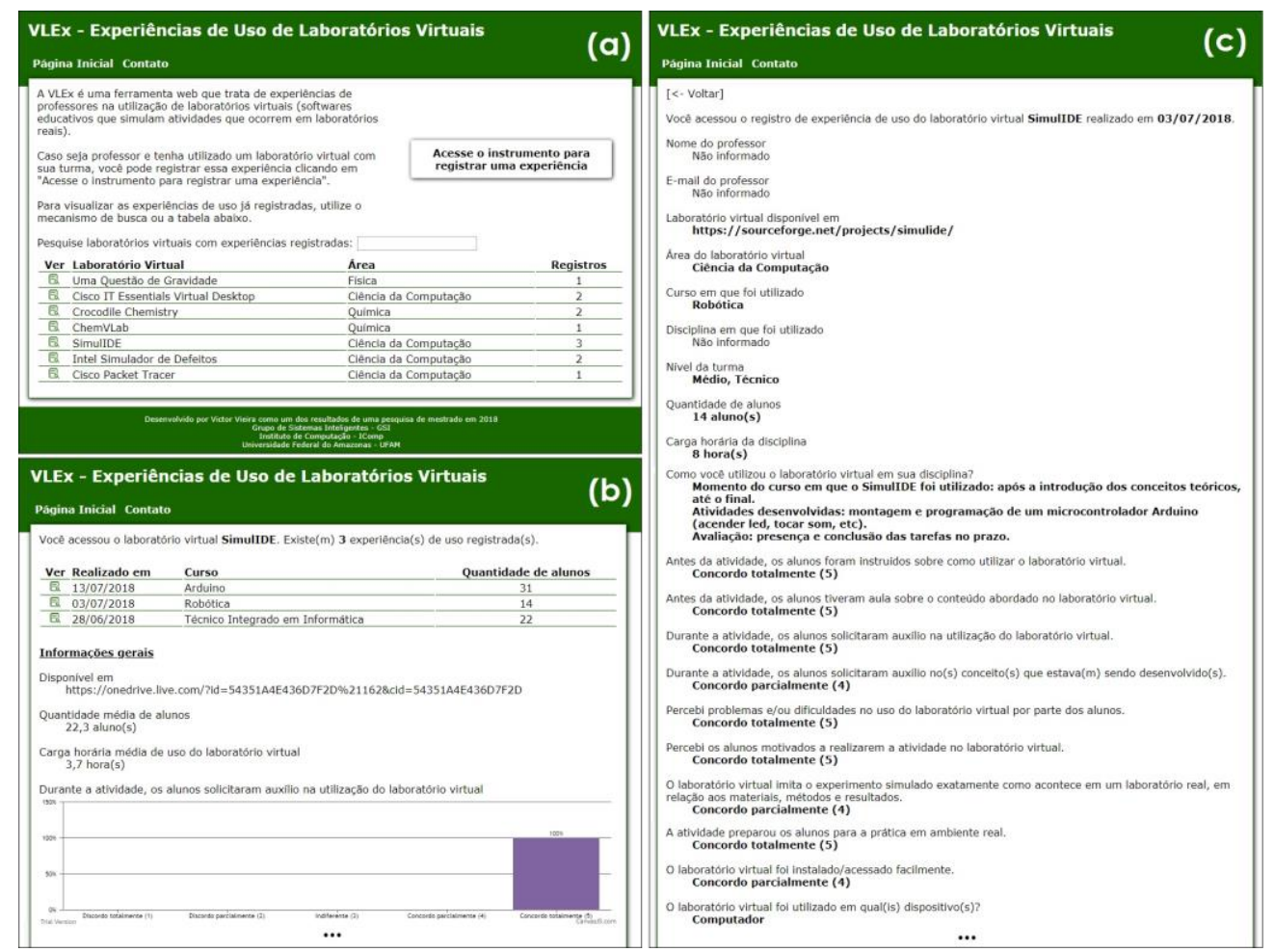

Figura 2. Telas da VLEx para exibição dos registros de experiência

A página inicial (a) concentra informações sobre toda a infraestrutura criada (instrumento e ferramenta), com uma descrição geral, link para o instrumento, um campo de busca e a relação dos LVs que possuem experiências de uso registradas, ordenados a partir do que possui o registro mais recente. Ao acessar um LV (b), o 
VII Congresso Brasileiro de Informática na Educação (CBIE 2018)

Anais do XXIX Simpósio Brasileiro de Informática na Educação (SBIE 2018)

usuário visualiza os registros realizados nele, também ordenados a partir do mais recente, com algumas informações sendo exibidas em porcentagem (gráfico) e médias. Com essa interface, espera-se que os usuários tenham uma visão geral sobre alguns dos aspectos relevantes do uso de determinado LV na perspectiva das várias experiências registradas. Uma visão completa de uma experiência usando um LV é obtida ao acessar um dos registros (c). Todas as respostas às questões do instrumento são exibidas de maneira organizada, facilitando a visualização e possibilitando uma análise detalhada. A ferramenta reflete detalhes importantes da experiência de um professor no uso de um LV com sua turma, registrados através da terceira versão do instrumento.

\section{Considerações finais}

O objetivo deste trabalho foi apresentar a evolução de um instrumento criado para registrar experiências de docentes no uso de laboratórios virtuais, que gerou uma infraestrutura composta pelo instrumento e uma ferramenta web para a exibição das experiências. Essa infraestrutura tem potencial para tornar úteis os dados geralmente não aproveitados de quando se termina uma atividade com um desses SEs, registrandoos e disponibilizando-os.

Os registros das experiências realizadas devem servir para auxiliar principalmente professores não acostumados a utilizar LVs no processo de seleção de um deles. O ponto de vista abordado no instrumento, que é o do docente, é ideal para auxiliar também na definição da metodologia a ser utilizada, a partir da adaptação de registros existentes. As informações registradas ainda tornam o instrumento relevante ao processo de avaliação dos LVs, mesmo que não seja esse o objetivo para o qual foi desenvolvido.

A infraestrutura desenvolvida cria novas possibilidades de pesquisas na área. Como trabalhos futuros, espera-se: a) avaliar a aceitação da infraestrutura criada; b) verificar quais os aspectos mais importantes para influenciar na seleção de LVs a partir dos resultados de uma experiência de uso; e c) gerar uma base de conhecimento com registros suficientes para o desenvolvimento de um sistema de recomendações de LVs.

\section{Referências}

Brito, A. e Cunha, J.. CloudLab: Um Ambiente Virtual de Aprendizagem com Laboratório Virtual Integrado para o Ensino de Hardware. Revista Brasileira de Informática na Educação, v20, n3. 2012.

Diwakar, S.; Radhamani, R.; Sasidharakurup, H.; Kumar, D.; Nizar, N.; Achuthan, K.; e Nair, B.. Assessing Students and Teachers Experience on Simulation and Remote Biotechnology VL: A Case Study with a Light Microscopy Experiment. 2016.

Frerich, S; Kruse, D.; Petermann, M.; e Kilzer, A.. Virtual Labs and Remote Labs: Practical experience for everyone, IEEE Global Engineering Education Conference (EDUCON), Istanbul. 2014.

Godoi, K. e Padovani, S.. Avaliação de material didático digital centrada no usuário: uma investigação de instrumentos passíveis de utilização. Produção, v19, n3. 2009.

Kalyvioti, K. e Mikropoulos, T.. A Virtual Reality Test for the Identification of Memory Strengths of Dyslexic Students in Higher Education. Journal of Universal Computer Science, v19, n18. 2013. 
VII Congresso Brasileiro de Informática na Educação (CBIE 2018)

Anais do XXIX Simpósio Brasileiro de Informática na Educação (SBIE 2018)

Kitchenham, B.; Charters, S.. Procedures for performing systematic literature reviews in software engineering. Keele University \& Durham University, UK. 2007.

Koh, J.. Designing and integrating reusable learning objects for meaningful learning: Cases from a graduate programme. Australasian Journal of Educational Technology. v33. 2017.

Lakatos, E.; e Marconi, M.. Fundamentos de Metodologia Científica. $8^{a}$ ed. São Paulo: Atlas. 2017.

Lucena, G.; dos Santos, V.; e da Silva, A.. Laboratório virtual como alternativa didática para auxiliar o ensino de química no ensino médio. Revista Brasileira de Informática na Educação, v21, n2. 2013.

Martins, V.; Abreu, F.; Militino, R.; Fukuoka, S.; e Guimarães, M.. Estratégia de Desenvolvimento, Implantação e Avaliação do uso da Realidade Virtual na Educação: Estudo de Caso na área de Português. Anais do $23^{\circ}$ Simpósio Brasileiro de Informática na Educação (SBIE 2012), Rio de Janeiro. 2012.

Nicolaci-Da-Costa, A. M.. O campo da pesquisa qualitativa e o Método de Explicitação do Discurso Subjacente (MEDS). Psicol. Ref. Crit., Porto Alegre, v20, n1. 2007.

Nunes, Felipe; Voss, Gleizer; Herpich, Fabrício; Sindeaux, Paulo; Tarouco, Liane; e Lima, José. Implementação e análise de um ambiente 3D para o ensino de Geografia. VI Congresso Brasileiro de Informática na Educação. Anais do XXVIII Simpósio Brasileiro de Informática na Educação. 2017.

Ramírez, J.; Rico, M.; Riofrío, D.; Berrocal-Lobo, M.; e de Antonio, A.. Students' Evaluation of a Virtual World for Procedural Training in a Tertiary-Education Course. Journal of Educational Computing Research. v56. 2017.

Seniow, K.; Nefcy, E.; Kelly, C.; e Koretsky, M.. Representations of student model development in virtual laboratories based on a cognitive apprenticeship instructional design. ASEE Annual Conference and Exposition, Conference Proceedings. 2010.

Vieira, V. e Gadelha, B.. RT-GSI-2018-0001. Instrumento para Registro de Experiência Docente no Uso de Laboratórios Virtuais - Primeira Versão. Relatório Técnico do Grupo de Sistemas Inteligentes. 2018. Disponível em https://goo.gl/UC3YJq.

Vieira, V. e Gadelha, B.. RT-GSI-2018-0002. Revisão Sistemática da Literatura Tecnologias para Registro de Experiência de Uso de Laboratórios Virtuais. Relatório Técnico do Grupo de Sistemas Inteligentes. 2018. Disponível em https://goo.gl/G72Zfl.

Vieira, V. e Gadelha, B.. RT-GSI-2018-0003. Instrumento para Registro de Experiência Docente no Uso de Laboratórios Virtuais - Segunda Versão. Relatório Técnico do Grupo de Sistemas Inteligentes. 2018. Disponível em https://goo.gl/pcw7xW.

Vieira, V. e Gadelha, B.. RT-GSI-2018-0004. Instrumento para Registro de Experiência Docente no Uso de Laboratórios Virtuais - Terceira Versão. Relatório Técnico do Grupo de Sistemas Inteligentes. 2018. Disponível em https://goo.gl/qVZv7b.

Yalcinalp, S. e Emiroglu, B.. Through efficient use of LORs: Prospective teachers' views on operational aspects of learning object repositories. British Journal of Educational Technology, v43. 2012. 